\title{
Uso de pruebas genómicas en tejido prostático, en el seguimiento de los pacientes con cáncer de próstata en protocolo de vigilancia activa: Revisión sistemática de la literatura
}

\section{Use of Genomic Evidence in Prostate Tissue in the Follow- up of Patients with Prostate Cancer under Active Surveillance Protocol: Systematic Literature Review}

\author{
Jorge Eduardo Gómez Meza ${ }^{1}$ Ana Carolina Lizcano \\ Daniel Rojas ${ }^{3}$ Juan Camilo García ${ }^{1}$ \\ 1 Urólogo/ Servicio de urología, Clínica Universitaria Colombia, \\ Bogotá D.C, Colombia \\ 2 Residente Urología/ Servicio de urología, Fundación Universitaria \\ Sanitas, Clínica Universitaria Colombia, Bogotá D.C, Colombia \\ 3 Urólogo oncólogo/ Jefe del servicio de Urología, Servicio de \\ urología, Clínica universitaria Colombia, Bogotá D.C, Colombia
}

\author{
Address for correspondence Jorge Eduardo Gómez Meza, \\ Urólogo, Clínica Universitaria Colombia, Bogotá, Colombia \\ (e-mail: jorgemeza99@gmail.com).
}

Urol Colomb 2018;27:132-140.

\section{Resumen \\ Palabras Claves \\ - pruebas genéticas \\ - cáncer de próstata \\ - marcadores genéticos \\ - vigilancia activa \\ - genómica}

Introducción y objetivos La vigilancia activa es una estrategia que busca diferir tratamientos con intención curativa y la morbilidad derivada de los mismos. Actualmente se requieren estrategias de seguimiento con mayor potencial de predicción en la enfermedad que permitan determinar de manera adecuada los pacientes candidatos para vigilancia activa. El objetivo de este estudio es realizar una revisión sistemática de la literatura sobre las pruebas genómicas en tejido prostático de los pacientes con cáncer de próstata en protocolo de vigilancia activa. Materiales y métodos Se realizó una revisión sistemática de la literatura basados en criterios de inclusión y exclusión, en las bases de datos Pubmed, Scopus, Embase y Cochrane, para pruebas genómicas en tejido prostático de los pacientes con cáncer de próstata en vigilancia activa, basados en el modelo PRISMA.

Resultados Los estudios incluidos tuvieron en cuenta los siguientes desenlaces: Progresión de la enfermedad, mortalidad cáncer específica, Gleason $>4$ y hallazgos adversos en la biopsia confirmatoria. Las pruebas más utilizadas fueron el TMPRSS2: ERG, alelos para riesgo de cáncer de próstata y polimorfismos simples de nucleótidos. Se encontró el ERG-2 como factor predictor de progresión de enfermedad. Para los polimorfismos simples de nucleótidos, la evidencia no mostró correlación con el hallazgo de Gleason >4, tiempo a tratamiento ni para hallazgos adversos en la biopsia confirmatoria, sin embargo, esos estudios fueron de carácter retrospectivo. Por el received

November 1, 2017

accepted

February 8, 2018

published online

April 9, 2018
DOI https://doi.org/

10.1055/s-0038-1639571.

ISSN 0120-789X.

eISSN 2027-0119.
Copyright (c) 2018, Sociedad Colombiana License terms de Urología. Publicado por Thieme Revinter Publicações Ltda., Rio de Janeiro, Brazil. Todos los derechos reservados.

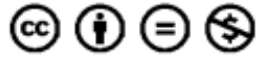


contrario, en otro estudio, se encontró un área bajo la curva de 0,66 para polimorfismos simples de nucleótidos lo que sugiere que ellos pueden discriminar entre un resultado favorable y desfavorable en la patología del espécimen. En cuanto a los alelos asociados al riesgo de características adversas en la patología, se encontró una asociación estadísticamente significativa y un aumento de dos veces mayor riesgo en aquellos pacientes que tuvieran la expresión de al menos 3 de esos alelos. Ninguna de las pruebas utilizadas en los diferentes estudios permitió tomar la decisión de continuar o suspender el protocolo de vigilancia activa.

Conclusión Los estudios sobre pruebas genómicas en tejido prostático de los pacientes con cáncer de próstata en vigilancia activa son escasos y con bajo poder estadístico, algunos de ellos sugieren la capacidad discriminatoria entre características favorables y desfavorables en la patología, sin embargo, esos resultados aún son controversiales y con poca validez interna. Se requieren de estudios prospectivos que permitan determinar el rol actual en el manejo de esos pacientes.

Abstract

\section{Keywords}

- genetic testing

- prostatic neoplasm

- genetic markers

- watchful waiting

- genomics
Introduction and Objectives Active surveillance (AS) is a strategy that aims postponing healing treatments and the morbidity resulting therefrom. Follow up strategies with a greater potential to predict the disease, which allow properly determining prospect patients for AS are required. The purpose of this study is a systematic review of the existing literature on prostatic tissue genomic tests (GT), in patients with prostate cancer (PC) under AS.

Material and Methods A systematic review of the literature was made in Pubmed, Scopus, Embase and Cochrane databases, following the PRISMA model.

Results The most used tests were TMPRSS2:ERG, alleles for PC risk and nucleotide simple polymorphism (NSP). ERG-2 was found as the predicting advance factor of the disease. For NSP, the evidence didn't show any correlation with the Gleason $>4$ findings, treatment period, or adverse findings in the confirming biopsy. On the other hand, an AUC: 0.66 for NSP was found, which suggests that can discriminate between favorable and unfavorable result. As to alleles associated to adverse features in the pathology, a statistically meaningful association was found and an increase of the risk in those patients that had a result of at least 3 of these. None of the tests allowed making the decision on whether to continue or not AS protocol.

Conclusion Studies are scarce and have a low statistical power; some of them suggest the discriminating ability between favorable and unfavorable characteristics in the pathology; however, these results are still controversial and have little internal validity.

\section{Introducción}

El cáncer de próstata (CaP) es la malignidad no cutánea, más común en el hombre. A pesar de su alta incidencia, la mortalidad cáncer especifica es baja. ${ }^{1}$ Uno de los factores relacionados con ese comportamiento, es el advenimiento del PSA, el cual desencadenó múltiples cambios en la enfermedad. Uno de los más importantes fue la migración de estadios avanzados a estadios localizados al momento del diagnóstico y la introducción del concepto de sobrediagnóstico definido epidemiológicamente como el cáncer que no será diagnosticado clínicamente a lo largo de la vida. Se estima que la tasa de sobrediagnóstico actual alcanza hasta el 56\% de los cánceres diagnosticados. ${ }^{2}$
Dentro de las opciones de tratamiento para paciente con CaP localizado de bajo riesgo, se encuentra la vigilancia activa (VA). Una estrategia de seguimiento que busca evitar la morbilidad asociada al tratamiento con intención curativa. Los pacientes son seguidos de forma sistemática con medición de PSA, realización de tacto rectal y biopsia transrectal de acuerdo al protocolo institucional. La decisión de retirar el paciente del protocolo y realizar tratamiento con intención curativa se basa en cambios en la biopsia de control, el PSA, aumento del volumen tumoral y la preferencia del paciente. ${ }^{3,4}$

Actualmente se busca encontrar pruebas que disminuyan el seguimiento invasivo en paciente en VA con pruebas genómicas en tejido prostático. Este artículo tiene como 
objetivo, realizar una revisión sistemática de la literatura, sobre el uso de esas pruebas en paciente con CaP en VA.

\section{Adquisición de la evidencia}

Se realizó una búsqueda sistemática de la literatura, basados en el Manual Cochrane de revisiones sistemáticas de intervenciones, versión 5.1.0 de marzo de $2011{ }^{2}$, realizando una búsqueda de artículos relevantes de forma electrónica en las bases de datos Pubmed, Embase, Scopus y Cochrane, utilizando las palabras claves: Pruebas genéticas; Cáncer de próstata; Marcadores genéticos; Vigilancia activa; Genómica. Se aplicaron los criterios de inclusión y exclusión, presentados en la -Tabla 1, para realizar la selección de los artículos siguiendo la metodología PRISMA (-Fig. 1). La búsqueda y selección de artículos se hizo por tres de los autores de forma individual coincidiendo en los artículos encontrados.

\section{Criterios de inclusión y exclusión}

Se incluyeron los estudios de tipo original, cohortes, casos y controles y experimentales en idioma inglés y español, publicados en los últimos 10 años, realizados en pacientes con cáncer de próstata en protocolo de vigilancia activa que evaluaran pruebas genómicas en tejido prostático. Se excluyeron aquellos estudios que estudiaran esas pruebas sin evidencia de malignidad, que fuesen realizadas en orina o en plasma, las series de casos, reporte de casos, comentarios editoriales y cartas al editor. A continuación, se presenta en la Tabla $\mathrm{N}^{\circ} 1$, un resumen de los criterios de inclusión y exclusión.
Tabla 1 Criterios de inclusión y exclusión

\begin{tabular}{|l|}
\hline Criterios de inclusión \\
\hline - Tipos de estudio: Artículos originales, estudios de \\
cohortes y casos y controles. \\
- Idioma de publicación de los artículos: inglés y \\
español. \\
- Fecha de publicación: Últimos 10 años (2007-2017). \\
- Condiciones especiales: Pacientes con cáncer de \\
próstata en protocolo de vigilancia activa. \\
\hline Criterios de exclusión \\
- Pacientes con patología prostática benigna. \\
- Estudios publicados en idiomas diferentes al inglés y \\
español. \\
- Artículos de revisión, series de casos, reporte de \\
casos, comentarios editoriales y cartas al editor. \\
- Artículos en muestras diferentes a tejido prostático. \\
\hline
\end{tabular}

\section{Extracción de datos}

Se realizó una agrupación de estudios inicialmente por título, posteriormente por las pruebas utilizadas en VA, teniendo en cuenta las pruebas estudiadas en cada estudio, su capacidad de predicción y el desenlace.

Luego de finalizar con la selección de los artículos, tres de los autores leyeron por completo cada uno de los artículos, con el fin obtener distintas opiniones y así tener la certeza de incluirlo o no en el estudio basados, principalmente, en si respondía o no la pregunta de investigación.

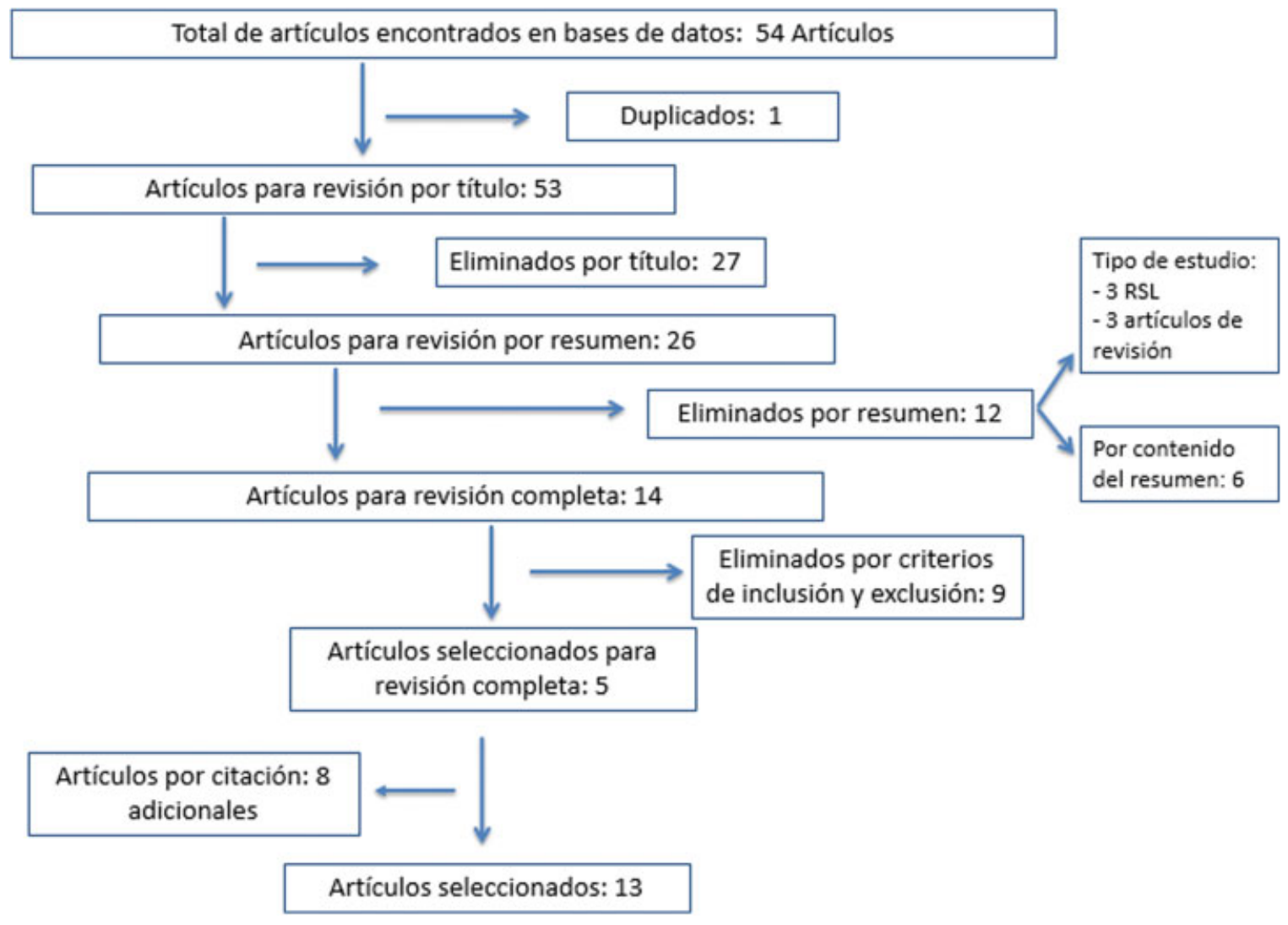

Fig. 1 Modelo PRISMA 


\section{Evaluación de Calidad de estudios y Sesgos}

Para determinar la calidad, limitaciones y sesgos de los estudios incluidos, se aplicó la declaración de la iniciativa STROBE (Strengthening the Reporting of Observational studies in Epidemiology) para la interpretación de los estudios observacionales.

\section{Resultados}

La búsqueda inicial arrojo 54 estudios. Se excluyó un artículo que se encontraba duplicado obteniendo un total de 53 estudios. Posteriormente se realizó una selección de artículos basado en los títulos obteniendo un total de 26 artículos. Se eliminaron 12 artículos; 6 por contenido en el resumen y 6 por ser estudios de tipo revisión narrativa y sistemática, obteniendo un total de 14 artículos para revisión completa, de los cuales se eliminaron 9 por no coincidir con los criterios de inclusión y exclusión, obteniendo un total de 5 artículos, con posterior inclusión por citación de 8 artículos, para un total de 13 artículos incluidos en esta revisión sistemática.

Se realizó una agrupación de estudios inicialmente por título, posteriormente por las pruebas utilizadas, su capacidad de predicción y el desenlace.

Los estudios incluidos en la revisión sistemática eran estudios observacionales (Estudios de cohortes 13), los cuales se publicaron en los últimos 10 años (2007- 2017); no se encontraron estudios de casos y controles como evidencia en la literatura sobre pruebas genómicas en tejido prostático. El número total de pacientes incluidos en los estudios fue de 5104 pacientes, siendo 111 el menor número de pacientes incluidos y el mayor de 1206 pacientes.

La prueba más frecuentemente evaluada fue el gen de fusión TMPRSS2:ERG, estudiada en 5 de los 13 artículos y el tejido prostático fue obtenido principalmente de biopsia transrectal de próstata (7/13 estudios), seguido de la patología obtenida de la resección transuretral de próstata.

Los desenlaces tenidos en cuenta en los estudios fueron: progresión de la enfermedad, mortalidad cáncer específica, patología adversa o progresión metastásica, tiempo al tratamiento, progresión del PSA y supervivencia global y/o cáncer especifica.

A continuación, se presenta la - Tabla 2 la cual compara los diferentes estudios incluidos.

\section{Resultados de evaluación de calidad y sesgos de los artículos incluidos}

Al aplicar la herramienta de evaluación descrita, se encontró que la totalidad de los artículos contaban con adecuada descripción de título y resumen, contexto y fundamentos, diseño del estudio, participantes, datos de las variables de resultado, resultados principales e interpretación de resultados.

Ninguno de los estudios describió cómo se calculó el tamaño de la muestra y la generabilidad. Tres de los trece estudios, carecían de objetivos en la introducción y de contexto en la metodología. Cinco estudios no reportaron la fuente de los datos y siete estudios no hicieron análisis de sesgos. En cuanto a la discusión de los artículos, uno de ellos no reportó los resultados claves, siete no reportaron limitaciones y tan solo cuatro estudios describen su estatus de financiación.

En la - Tabla 3, se presenta un resumen de la aplicación de la declaración de la iniciativa STROBE.

\section{Limitaciones}

Dentro de las principales limitaciones de los estudios encontramos las siguientes, presentadas en la -Tabla 4.

\section{Discusión}

Las estrategias de seguimiento de los pacientes con cáncer de próstata en vigilancia activa, incluyen pruebas invasivas, mórbidas y que afectan la calidad de vida. El advenimiento de las pruebas genómicas en tejido prostático, surge como una estrategia innovadora y con mayor capacidad de predicción del curso de la enfermedad.

De los estudios revisados, uno de los marcadores genéticos más estudiados en tejido prostático fue el gen fusión TMPRSS2: ERG. En la mayoría de los estudios, se concluye que la presencia del gen fusión TMPRSS2:ERG, se asocia con predicción de supervivencia global, mortalidad cáncer específica y riesgo de progresión de la enfermedad. ${ }^{5,6}$ En un artículo publicado en el 2007, ${ }^{7}$ se encontró que los pacientes con tumores con expresión del gen fusión TMPRSS2:ERG, presentaban una mayor puntuación de Gleason y una asociación estadísticamente significativa con la presencia de enfermedad metastásica y menor supervivencia cáncer específica.

Berg y cols., encontraron una asociación entre los pacientes con ERG positivos y progresión de la enfermedad, considerando como posible explicación, que el fenotipo agresivo es secundario a la proliferación de los genes de expresión, sin embargo, esos resultados no fueron estadísticamente significativos. De la misma manera, Sboner y cols, encontraron la expresión de ERG, como factor predictor de patología adversa letal ( $\mathrm{OR}=7,2$ $95 \% \mathrm{CI}=[2,3 ; 19,0]) .^{8-10}$

La mayoría de esos estudios se asocian con un mayor riesgo de progresión, pero ninguno de los artículos incluidos en esta revisión coincide con que los pacientes ERG-positivos deban ser excluidos de los protocolos de vigilancia activa. ${ }^{8}$

En cuanto a los paneles genéticos, son pocos los estudios y los resultados son controversiales. En el estudio de Sboner y cols., se encontró un AUC de 0,75 para los genes estudiados y para las variables clínicas de la patología y el Gleason, por lo que no es posible recomendar el estudio de genes en la muestra de tejido prostático. ${ }^{9}$

En la publicación realizada por Penney y cols. en el 2011, se analizaron 157 genes para predecir la subestadificación del puntaje de Gleason; encontrando un AUC de 0,91 en la cohorte Sueca y un AUC de 0,94 en la cohorte del PHS, concluyendo que la aplicación de esos paneles permite predecir la presencia de enfermedad letal sin la posibilidad de discriminar entre el Gleason $4+3$ versus $3+4$ (P 0,006). ${ }^{11}$

Existen múltiples test de expresión génica, como son ConfirmMDx, Prolaris, OncotypeDx, Decipher, de esos con aplicabilidad en vigilancia activa se encuentran el Prolaris y el OncotypeDx. En el 2014, Klein y cols, validaron la prueba de OncotypeDx, encontrando que de 732 genes: 282 (39\%) 


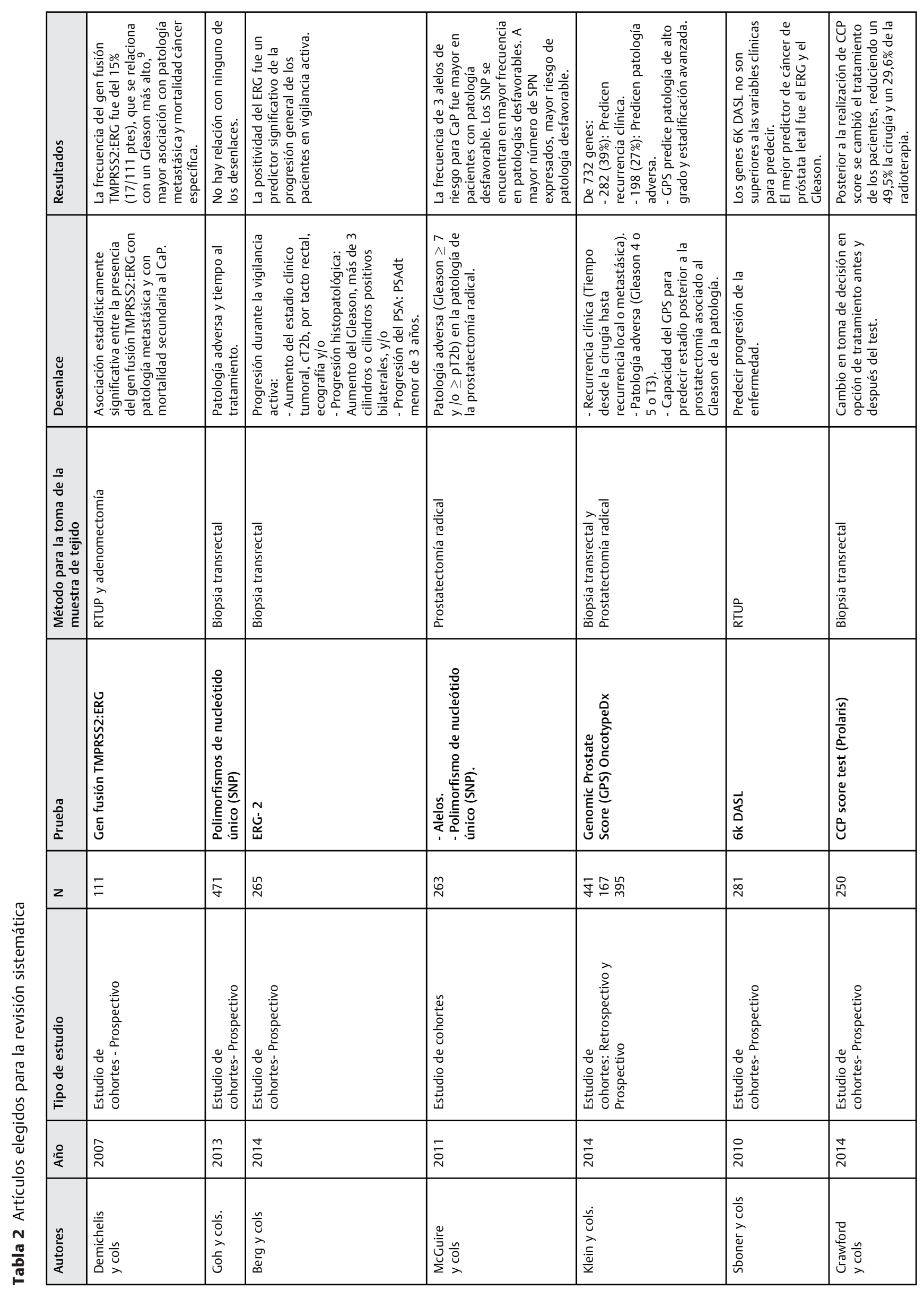




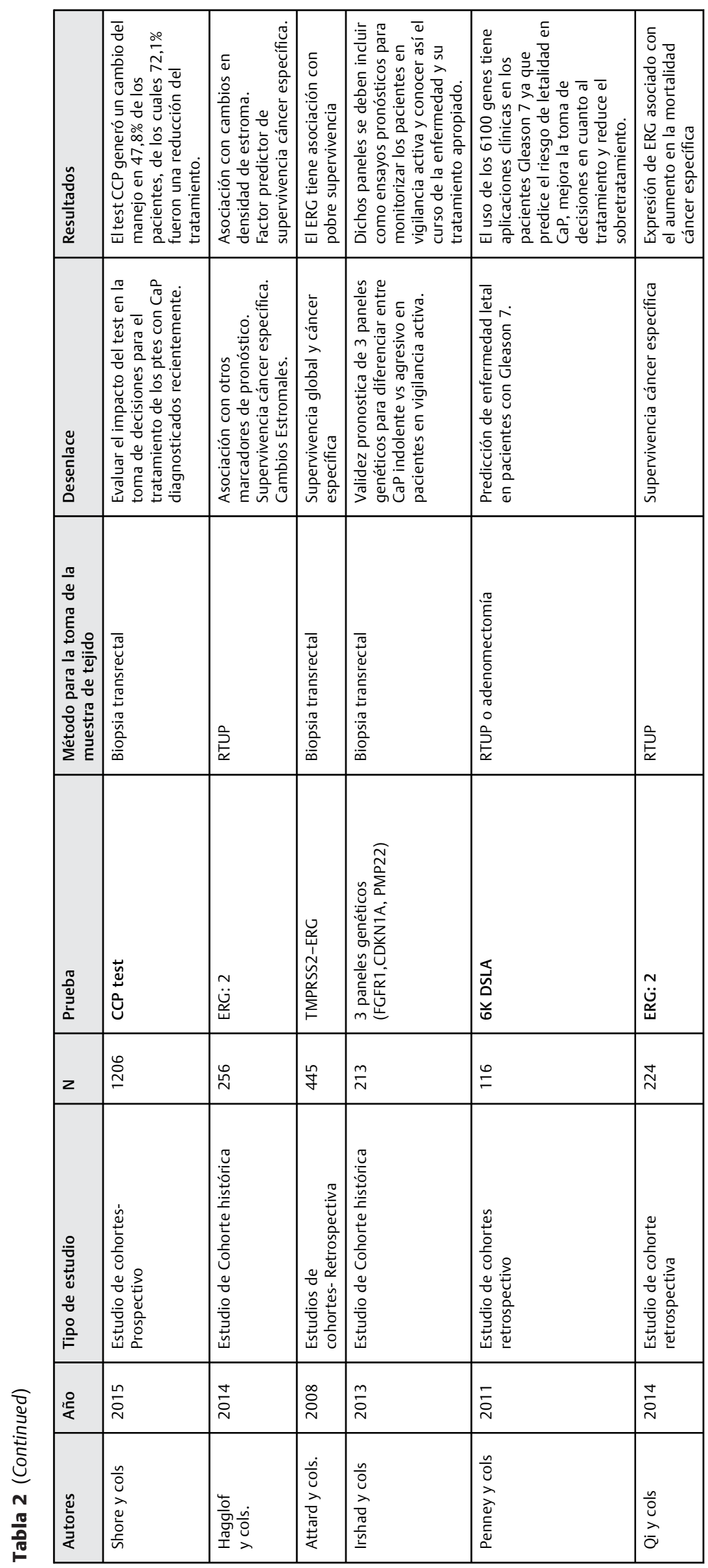




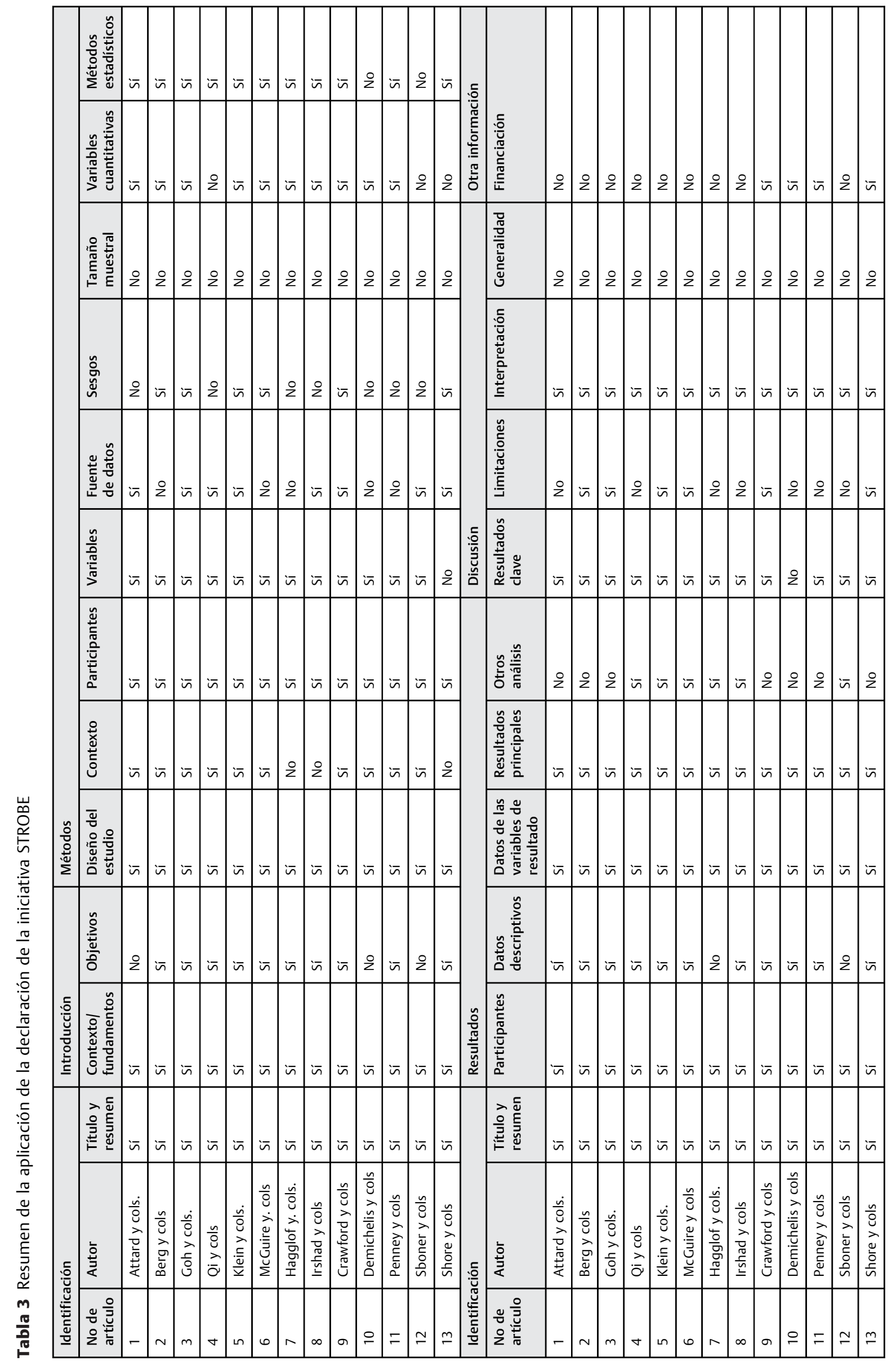


Tabla 4 Limitaciones de los estudios

\begin{tabular}{|c|c|}
\hline Autores & Limitaciones \\
\hline Demichelis y cols & No describen limitaciones. \\
\hline Goh y cols. & $\begin{array}{l}\text { - Pequeño tamaño de la muestra con un seguimiento relativamente corto en la cohorte de CaP de bajo } \\
\text { riesgo. } \\
\text { - El estudio no consideró el impacto del medio ambiente y cambios en la calidad de vida de los pacientes } \\
\text { posterior al diagnóstico de CaP, los cuales son factor protector en cohortes de vigilancia activa. } \\
\text { - Aún falta descubrir el genoma completo del CaP, lo cual cambiaría el cálculo de los puntajes de riesgo y } \\
\text { mejoraría su capacidad predictiva. }\end{array}$ \\
\hline Berg y cols & $\begin{array}{l}\text { - Limitaciones de naturaleza observacional. } \\
\text { - Se incluyeron pacientes que no cumplían estrictamente con los criterios de vigilancia activa. } \\
\text { - Se desconocía la supervivencia, desenlace e histopatología de los pacientes llevados a prostatectomía } \\
\text { radical con ERG positivo. }\end{array}$ \\
\hline McGuire y cols & $\begin{array}{l}\text { - Tamaño de la muestra insuficiente. } \\
\text { - Todos los hombres fueron tratados con PR y ninguno se inscribió en un protocolo de VA, el estudio sufre } \\
\text { de sesgo de selección y puede no ser universalmente aplicable a los hombres que se someten a } \\
\text { vigilancia activa. } \\
\text { - Se desconoce la progresión de los pacientes si hubieran sido llevados a vigilancia activa. } \\
\text { - Al examinar los } 35 \text { SNP, aumenta la probabilidad de errores tipo } 1 \text { cuando la } p<0.05 \text { y al aplicar la } \\
\text { corrección de Bonferroni, ninguno de los SNP tiene significancia estadística. }\end{array}$ \\
\hline Klein y cols. & $\begin{array}{l}\text { - Se analizaron } 732 \text { genes mediante PCR y no se utilizaron microarrays ni otras nuevas tecnologías. } \\
\text { - Se analizaron los genes en muestras de ptes con CaP localizado más no en pacientes con CaP } \\
\text { metastásico para medir la agresividad. } \\
\text { - Solo se incluyeron pacientes con CaP con Gleason } 3+4 \text {, no patrón de Gleason } 4 \text { como primario ni } \\
\text { Gleason } 5 \text {. }\end{array}$ \\
\hline Sboner y cols & No describe limitaciones. \\
\hline Crawford y cols & $\begin{array}{l}\text { - El registro de los pacientes permitió que cualquiera fuera incluido y por lo tanto es posible que el clínico } \\
\text { haya ordenado la prueba solamente para los pacientes para los cuales él consideró pertinente realizar la } \\
\text { evaluación de riesgo más precisa, llevando a una sobreestimación de la influencia clínica de la prueba. } \\
\text { - El estudio no evaluó específicamente el rol de los pacientes en cuanto a la elección terapéutica, por lo } \\
\text { que se considera que se debe hacer una evaluación más personalizada del riesgo que permita que el } \\
\text { paciente participe activamente en la decisión terapéutica. }\end{array}$ \\
\hline Shore y cols & $\begin{array}{l}\text { - El uso de diagnóstico molecular para establecer el riesgo en CaP está recientemente incorporado y los } \\
\text { autores aún no tienen experiencia en el tema. } \\
\text { - La toma de la decisión del cambio del tratamiento solo se basó en los resultados del test CCP, sin tener } \\
\text { en cuenta otros factores contributivos. }\end{array}$ \\
\hline Hagglof y cols. & No describe limitaciones. \\
\hline Attard y cols. & No describe limitaciones. \\
\hline Irshad y cols & No describe limitaciones. \\
\hline Penney y cols & No describe limitaciones. \\
\hline Qi y cols & No describe limitaciones \\
\hline
\end{tabular}

predicen recurrencia clínica y 198 genes (27\%) predicen patología adversa, logrando así, escoger 17 genes para la validación de la prueba GPS, la cual predice patología adversa y estadificación avanzada, por lo que pueden ser útiles en protocolos de vigilancia activa. ${ }^{12}$ Adicionalmente, estudios como el de Crawaford y cols. y Shore y cols., en 2014 y 2015 respectivamente, mostraron que el uso de Prolaris, cambió la conducta del tratamiento, evidenciando una importante reducción en manejo con intención curativa, 49,5\% de cirugía, $29,6 \%$ de radioterapia en el estudio de Shore y un cambio en la conducta de tratamiento $47,8 \%$, incluyendo tanto cirugía como radioterapia, en el estudio de Crawford. ${ }^{13,14}$

En cuanto a los alelos de riesgo para cáncer de próstata, los resultados entre los diferentes estudios han sido muy controversiales. El único estudio prospectivo fue realizado por McGuire y cols. en el 2011, ${ }^{15}$ quienes refieren una mayor asociación entre 3 alelos específicos de riesgo para CaP: (rs1447295 [8q24], rs1571801 [9q33.2] y rs11228565 [11q13]) y hallazgos desfavorables en la patología definitiva con valores estadísticamente significativos, $p=0,004$, $p=0,03$ y $p=0,02$ respectivamente, concluyendo que los portadores de uno de estos alelos, tienen un riesgo 2 veces mayor de presentar características desfavorables en la patología $(p=0.03)$ y que ser portador de 2 alelos, aumenta 7 veces más el riesgo $(p=0,001)$.

Adicionalmente, éste estudio evidenció la capacidad que tienen los polimorfismos simples de nucleótidos para discriminar entre patología favorable y desfavorable con un AUC de 0,66, sin embargo, al compararlo con otras herramientas de predicción en vigilancia activa, como la resonancia magnética, el AUC, no fue superior a esta $(0,83){ }^{16}$ Igualmente, Goh y cols ${ }^{17}$ encontraron que los 
resultados no tienen suficiente significancia estadística para recomendar el uso de esos polimorfismos en los protocolos de vigilancia activa.

En cuanto a las limitaciones encontramos que en los estudios incluidos, no hay una adecuada rigurosidad metodológica, hay heterogeneidad en los desenlaces, en la presentación de resultados y en los criterios de inclusión en vigilancia activa, que se traduce en una limitada validez interna y que no hace posible realizar una comparación cuantitativa.

\section{Conclusiones}

Los estudios sobre pruebas genómicas en tejido prostático de pacientes con cáncer de próstata en protocolo de vigilancia activa son escasos y con bajo poder estadístico; algunos de ellos sugieren la capacidad discriminatoria entre características favorables y desfavorables en la patología, sin embargo, esos resultados aún son controversiales y con poca validez interna. Es por eso que se requieren estudios prospectivos que permitan determinar el rol actual del manejo en esos pacientes.

Nota

No hay agradecimientos. No hay conflictos de interés.

Protección de personas y animales

Los autores declaran que para esta investigación no se han realizado experimentos en seres humanos ni en animales.

Confidencialidad de los datos

Los autores declaran que en este artículo no aparecen datos de pacientes.

Derecho a la privacidad y consentimiento informado Los autores declaran que en este artículo no aparecen datos de pacientes.

\section{Bibliografía}

1 Shukla ME, Yu C, Reddy CA, et al. Evaluation of the current prostate cancer staging system based on cancer-specific mortality in the surveillance, epidemiology, and end results database. Clin Genitourin Cancer 2015;13(01):17-21

2 Bastian PJ, Carter BH, Bjartell A, et al. Insignificant prostate cancer and active surveillance: from definition to clinical implications. Eur Urol 2009;55(06):1321-1330
3 Leapman MS, Ameli N, Cooperberg MR, et al. Quantified Clinical Risk Change as an End Point During Prostate Cancer Active Surveillance. Eur Urol 2017;72(03):329-332

4 Lin DW, Newcomb LF, Brown MD, et al; Canary Prostate Active Surveillance Study Investigators. Evaluating the Four Kallikrein Panel of the 4Kscore for Prediction of High-grade Prostate Cancer in Men in the Canary Prostate Active Surveillance Study. Eur Urol 2017;72(03):448-454

5 Attard G, Clark J, Ambroisine L, et al; Transatlantic Prostate Group. Duplication of the fusion of TMPRSS2 to ERG sequences identifies fatal human prostate cancer. Oncogene 2008;27(03):253-263

6 Hägglöf C, Hammarsten P, Strömvall K, et al. TMPRSS2-ERG expression predicts prostate cancer survival and associates with stromal biomarkers. PLoS One 2014;9(02):e86824. Doi: 10.1371/ journal.pone.0086824

7 Demichelis F, Fall K, Perner S, et al. TMPRSS2:ERG gene fusion associated with lethal prostate cancer in a watchful waiting cohort. Oncogene 2007;26(31):4596-4599

8 Berg KD, Vainer B, Thomsen FB, et al. ERG protein expression in diagnostic specimens is associated with increased risk of progression during active surveillance for prostate cancer. Eur Urol 2014;66(05):851-860. Doi: 10.1016/j.eururo.2014.02.058 [Internet]

9 Sboner A, Demichelis F, Calza S, et al. Molecular sampling of prostate cancer: a dilemma for predicting disease progression. BMC Med Genomics 2010;3:8

10 Qi M, Yang X, Zhang F, et al. ERG rearrangement is associated with prostate cancer-related death in Chinese prostate cancer patients. PLoS One 2014;9(02):e84959. Doi: 10.1371/journal.pone.0084959

11 Penney KL, Sinnott JA, Fall K, et al. mRNA expression signature of Gleason grade predicts lethal prostate cancer. J Clin Oncol 2011; 29(17):2391-2396

12 Klein EA, Cooperberg MR, Magi-Galluzzi C, et al. A 17-gene assay to predict prostate cancer aggressiveness in the context of Gleason grade heterogeneity, tumor multifocality, and biopsy undersampling. Eur Urol 2014;66(03):550-560

13 Shore ND, Kella N, Moran B, et al. Impact of the Cell Cycle Progression Test on Physician and Patient Treatment Selection for Localized Prostate Cancer. J Urol 2016;195(03):612-618

14 Crawford ED, Scholz MC, Kar AJ, et al. Cell cycle progression score and treatment decisions in prostate cancer: results from an ongoing registry. Curr Med Res Opin 2014;30(06):1025-1031

15 Mcguire BB, Helfand BT, Kundu S, et al. Association of prostate cancer risk alleles with potential candidates for active surveillance. BJU Int 2012;110(03):338-343

16 van As NJ, Norman AR, Thomas K, et al. Predicting the probability of deferred radical treatment for localised prostate cancer managed by active surveillance. Eur Urol 2008;54(06):1297-1305

17 Goh CL, Saunders EJ, Leongamornlert DA, et al. Clinical implications of family history of prostate cancer and genetic risk single nucleotide polymorphism (SNP) profiles in an active surveillance cohort. BJU Int 2013;112(05):666-673 